\title{
Correspondence:
}

\section{Efficacy of internet-based integrated intervention on depression and anxiety symptoms in patients with COVID-19*}

\author{
Ning WEI ${ }^{1,2,3}$, Bo-chao HUANG ${ }^{1,2,3}$, Shao-jia LU ${ }^{1,2,3}$, \\ Jian-bo HU ${ }^{1,2,3}$, Xiao-yi ZHOU ${ }^{1,2,3}$, Chan-chan $\mathrm{HU}^{1,2,3}$, \\ Jing-kai CHEN ${ }^{1,2,3}$, Jin-wen HUANG ${ }^{1,2,3}$, Shu-guang \\ $\mathrm{LI}^{1,2,3}$, Zheng WANG ${ }^{1,2,3}$, Dan-dan $\mathrm{WANG}^{1,2,3}$, \\ Yi XU ${ }^{\dagger 1,2,3}$, Shao-hua HU ${ }^{\dagger 1,2,3}$ \\ ${ }^{1}$ Department of Psychiatry, the First Affiliated Hospital, School of \\ Medicine, Zhejiang University, Hangzhou 310003, China \\ ${ }^{2}$ The Key Laboratory of Mental Disorder's Management of Zhejiang \\ Province, Hangzhou 310003, China \\ ${ }^{3}$ Zhejiang Engineering Center for Mathematical Mental Health, \\ Hangzhou 310003, China \\ †E-mail: xuyizju@zju.edu.cn; dorhushaohua@zju.edu.cn
}

https://doi.org/10.1631/jzus.B2010013

Published online Apr. 1, 2020

Public health crises, such as the outbreak of severe acute respiratory syndrome coronavirus 2 (SARSCoV-2) since Dec. 2019, are widely acknowledged as severe traumatic events that impose threats not only because of physical concerns but also because of the psychological distress of infected patients. We designed an internet-based integrated intervention and evaluated its efficacy on depression and anxiety symptoms in patients infected by SARS-CoV-2.

Wuhan, the capital city of Hubei Province, China, has attracted global attention because of the outbreak of SARS-CoV-2-infected pneumonia (coronavirus disease 2019 (COVID-19)). On Jan. 3,

\footnotetext{
${ }^{\ddagger}$ Corresponding authors

* Project supported by the Science and Technology Department of Zhejiang Province (No. 2017C37037), the National Key Research and Development Program of China (Nos. 2016YFC1307100 and 2018YFC1314200), the Zhejiang Provincial Natural Science Foundation of China (Nos. LQ20H090012 and LQ20H090010), and the Zhejiang University Special Scientific Research Fund for COVID-19 Prevention and Control (No. 2020XGZX046), China

(ib) ORCID: Yi XU, https://orcid.org/0000-0002-8773-8264; Shao-hua HU, https://orcid.org/0000-0003-0570-670X

(C) Zhejiang University and Springer-Verlag GmbH Germany, part of Springer Nature 2020
}

2020, the SARS-CoV-2 was isolated in samples of broncho-alveolar lavage fluid from a patient in $\mathrm{Wu}-$ han and was confirmed as the cause of the novel CoV-infected pneumonia (Zhu et al., 2020). On Jan. 20, 2020, Chinese experts confirmed human-to-human transmission of SARS-CoV-2 and infection of medical staff. Three days after that, the Chinese government locked down Wuhan in an unprecedented effort to curb the spread of SARS-CoV-2 (Xinhua Net, 2020). The SARS-CoV-2 infection has been reported not only in China, but also in other countries. As of Mar. 12, 2020, the number of confirmed cases of the novel CoV approached 128000 with a total of 4713 reported deaths in at least 118 countries.

In the fight against the SARS-CoV-2, COVID19 patients have been experiencing enormous psychological pressure, specifically including a lack of knowledge about the consequences of infection by a potentially fatal new virus, isolation, and feelings of helplessness. These situations are giving rise to mental health problems, such as stress, anxiety, depressive symptoms, insomnia, and fear in patients with confirmed SARS-CoV-2. Moreover, symptoms of the infection, such as fever, fatigue, and dyspnea, as well as the side effects of treatments, could result in worsening mental distress (Xiang et al., 2020). Consistently, in the early stage of the SARS outbreak, the emergency of which had parallels with the COVID-19 epidemic, a range of psychiatric conditions, including high levels of stress, depressive symptoms, anxiety, insomnia, nightmares, and poor concentration, were reported by SARS patients (Chua et al., 2004). Further evidence suggested that these psychiatric morbidities might be still significant even when persons infected with SARS recovered physically (Tsang et al., 2004), and SARS survivors who had a history of psychiatric consultation in the acute phase of the illness had a higher risk for psychological distress 
later, after discharge from the hospital, and might warrant timely and focused mental health support services (Wu et al., 2005).

Although data from systematic studies of SARSCoV-2 do not yet exist, evidence suggests that this disease has psychosocial consequences for COVID-19 patients (Duan and Zhu, 2020). The psychological impacts of SARS-CoV-2 should not be overlooked. However, evidence-based interventions targeted at COVID-19 patients are scarce. Thus, the aims of the present study are to introduce an internet-based integrated intervention to COVID-19 patients with psychological distress and to evaluate the efficacy of the provided mental health care on depression and anxiety symptoms in patients with COVID- 19 .

This is a prospective, randomized, controlled, 2-week study conducted in the First Affiliated Hospital, School of Medicine, Zhejiang University (Hangzhou, China) from Feb. 2 to Feb. 28, 2020. All the laboratoryconfirmed COVID-19 patients in the isolation ward were screened by Patient Health Questionnaire-9 (PHQ-9) (Kroenke et al., 2001) and Generalized Anxiety Disorder-7 (GAD-7) (Spitzer et al., 2006) for psychological distress and finally, a total of 26 COVID-19 patients were enrolled in this study. The inclusion criteria were as follows: (1) aged 18-65 years; (2) PHQ-9 or GAD-7 of $\geq 5$; (3) completed at least a junior middle school level of education. Any participant who met one of the following criteria was excluded: (1) PHQ-9 or GAD-7 of $\geq 15$; (2) with suicidal ideation; (3) use of any antipsychotics; (4) the underlying disease was too severe to complete the assessments; (5) unable to follow the instructions of the internet-based intervention. A list of random sequence assignments for each group was drawn up by computer at the beginning of the study. After recruitment, 26 participants were randomly assigned to groups sequentially, with an equal probability of receiving an internet-based integrated intervention (intervention group) or just supportive care (control group), with 13 participants in each group. The 17-item Hamilton Depression Scale (17-HAMD) (Hamilton, 1967) and Hamilton Anxiety Scale (HAMA) (Hamilton, 1959) were used to assess the severity of depression and anxiety symptoms, respectively, and the efficacy of the internet-based integrated intervention every week. The outcomes of psychological assessments were analyzed by repeated measures analyses of variance with time as the within-subjects factor and group as the between-subjects factor. Last observation carried forward (LOCF) analysis was performed only in patients who dropped out and failed to complete two-week intervention.

The internet-based integrated intervention is a self-help intervention containing four main components: breath relaxation training, mindfulness (body scan), "refuge" skills, and butterfly hug method. The instructions for this internet-based integrated intervention were audio-recorded and then put online. All subjects in the intervention group were guided to listen to the audio via their mobile phones and follow the instructions for intervention training at a fixed time every day for two weeks. It would take the patient about $50 \mathrm{~min}$ to finish this everyday task. In addition, during the study period, the participants in the control group received daily supportive care only.

As indicated in Table 1, nine (34.6\%) of the 26 patients had underlying diseases: four (15.4\%) had hypertension, two $(7.7 \%)$ had liver disease, and one (3.8\%) each had gastric ulcer, coronary heart disease (CHD), and acquired immune deficiency syndrome (AIDS). No significant difference between the two groups was observed for age, gender, severity of illness, baseline scores of PHQ-9, GAD-7, 17-HAMD, or HAMA. Three patients in the intervention group and one patient in the control group failed to complete the assessments at the end of the second week because of discharge from hospital. Overall, there was a significant time effect indicating reduction in mean scores of 17-HAMD $(F=37.35, P<0.001)$ and HAMA $(F=26.58, P<0.001)$, as well as a main effect of group (17-HAMD: $F=4.384, P=0.047$; HAMA: $F=5.634$, $P=0.026)$ and a group-by-time interaction (17-HAMD: $F=5.268, P=0.009$; HAMA: $F=3.743, P=0.031)$. There were no main or interactive effects of age, gender, or severity of illness on scores of 17-HAMD and HAMA when introduced as covariates in the analyses. Post hoc analyses of the individual time points showed that scores of 17-HAMD and HAMA were significantly decreased in patients of the intervention group at the end of the first (17-HAMD, $t=-2.381, P=0.026$; HAMA, $t=-2.263, P=0.033)$ and second (17-HAMD, $t=-3.089, P=0.005$; HAMA, $t=-3.746, P=0.001)$ weeks, when compared with the patients of the control group (Fig. 1).

In this study, we designed an internet-based integrated intervention focusing on relaxation, selfcare, and raising the sense of security. To the best of 
Table 1 Demographic and clinical characteristics of all subjects $(n=26)$

\begin{tabular}{lcccc}
\hline \multicolumn{1}{c}{ Characteristics } & Intervention group $(n=13)$ & Control group $(n=13)$ & $t / \chi^{2}$ & $P$-value \\
\hline Age (year) & $40.8 \pm 13.5$ & $48.5 \pm 9.5$ & $t=-1.693$ & 0.103 \\
Gender (male/female) & $9 / 4$ & $7 / 6$ & $\chi^{2}=0.650$ & 0.420 \\
Severity of illness & & & & \\
$\quad$ Medium & $5(38.5)$ & $4(30.8)$ & & 0.680 \\
$\quad$ Severe & $8(61.5)$ & $9(69.2)$ & $\chi^{2}=0.170$ & \\
Coexisting conditions & & & & \\
Any & $5(38.5)$ & $4(30.8)$ & & \\
Hypertension & $3(23.1)$ & $1(7.7)$ & & \\
Live disease & $1(7.7)$ & $1(7.7)$ & & \\
Gastric ulcer & $0(0.0)$ & $0(0.0)$ & & \\
CHD & $1(7.7)$ & $1(7.7)$ & & \\
AIDS & $0(0.0)$ & $7.76 \pm 2.89$ & $t=-0.681$ \\
PHQ-9 score & $7.08 \pm 2.25$ & $5.92 \pm 2.53$ & $t=-0.816$ & 0.423 \\
GAD-7 score & $5.08 \pm 2.75$ & $12(92.3)$ & $\chi^{2}=0.000$ & 1.000 \\
PHQ-9 score $\geq 5$ & $12(92.3)$ & $10(76.9)$ & $\chi^{2}=2.600$ & 0.107 \\
GAD-7 score $\geq 5$ & $6(46.2)$ & & & \\
\hline
\end{tabular}

Data were expressed as number, number (percentage), or mean \pm standard deviation (SD). CHD: coronary heart disease; AIDS: acquired immune deficiency syndrome; PHQ-9: Patient Health Questionnaire-9; GAD-7: Generalized Anxiety Disorder-7

(a)

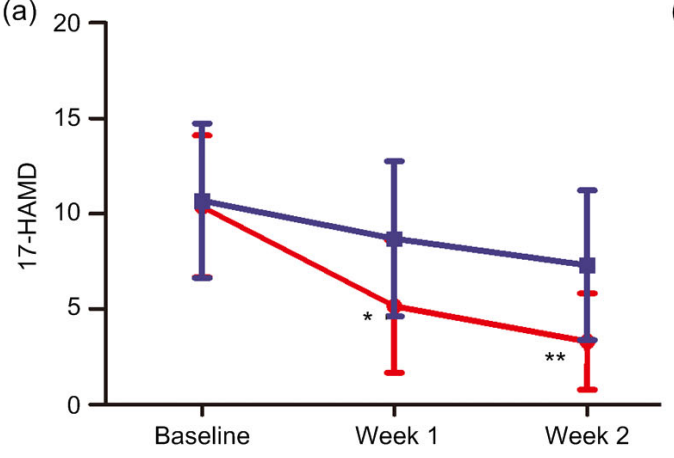

(b)

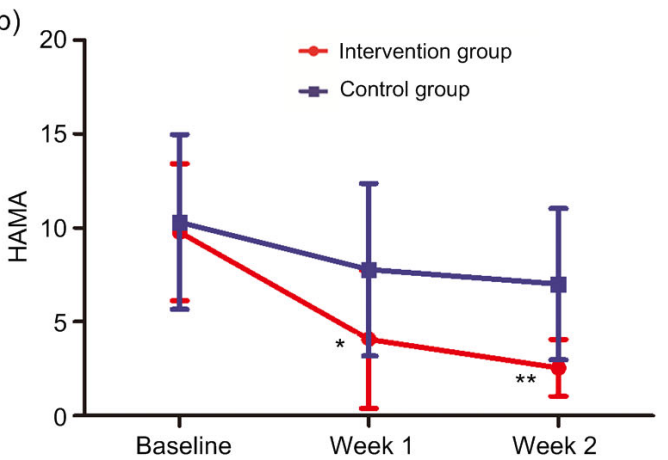

Fig. 1 Changes in scores of 17-HAMD and HAMA across the experimental groups

(a) Significant decreases of scores of 17-HAMD were observed in patients of the intervention group at the end of the first ( $t=-2.381, P=0.026)$ and second $(t=-3.089, P=0.005)$ weeks when compared with the patients of the control group since recruitment. (b) Consistent with scores of 17-HAMD, as compared with the controls, patients of the intervention group showed decreased scores of HAMA with statistical significance at the end of the first $(t=-2.263, P=0.033)$ and second $(t=-3.746, P=0.001)$ weeks since recruitment. 17-HAMD, 17-item Hamilton Depression Scale; HAMA, Hamilton Anxiety Scale. ${ }^{*} P<0.05,{ }^{* *} P<0.01$, compared with the control group $(n=13)$. Data are expressed as mean \pm standard deviation (SD)

our knowledge, it is the first time that the efficacy of this internet-based self-help intervention has been evaluated for COVID-19 patients with psychological distress. We found that patients of the intervention group exhibited significantly decreased levels of depression and anxiety symptoms in comparison with those of the control group, indicating that our internetbased integrated intervention showed a rapid improvement on mood disturbance and it should be applied in the management of psychological distress in COVID-19 patients.
To date, the popularity of internet services and smartphones has enabled mental health professionals and health authorities to provide online mental health services (Liu et al., 2020). It has been demonstrated in a previous meta-analysis that internet-delivered cognitive behavior therapy (ICBT) was as effective as face-to-face treatment for depressive symptoms, social anxiety disorder, and other psychological or somatic disorders (Andersson et al., 2014). In this context, internet-based interventions increasingly complement face-to-face counseling or therapy. Furthermore, 
internet-based interventions also showed advantages over face-to-face therapy, and the benefits mainly included low threshold accessibility, flexible use, independence of time and place, use at a selfdetermined pace, a high level of autonomy and privacy, and lower costs (Schröder et al., 2016). These factors might be especially relevant for COVID-19 patients since the human-to-human transmission of SARS-CoV-2 was confirmed and the infected patients were in quarantine. Internet-based intervention could also diminish the risk of infection in mental healthcare providers.

Consistent with our findings, prior evidence has been found for the benefit of internet-based intervention programs on mental health, particularly for depression, stress and distress, and anxiety. The types of interventions that predominated as efficacious included information and education with or without professional psychological support, and, to a lesser extent, combined with peer and psychological support (Sherifali et al., 2018). Nevertheless, in an internetbased intervention comprising five structured confrontational writing assignments, for individuals who experienced the death of a first-degree relative and who were significantly distressed, although writing decreased feelings of emotional loneliness and increased positive mood, writing did not affect grief or depressive symptoms. One possible explanation for the unexpected outcome might be related to the fact that the participants who entered the study were on average quite distressed (van der Houwen et al., 2010). In our study, only patients with mild to moderate depression or anxiety symptoms were recruited, and the results were in accord with our initial expectation. Taken together, the current findings provided information that individuals with mild to moderate psychological distress might be more suitable for internetbased intervention.

The results of the present study should be interpreted in the light of several limitations. First, the sample size of each group was relatively small, which limited its statistical power to detect real effects. Secondly, this study might have a risk of bias for blinding of outcome assessments, because the evaluation was also performed by the intervention provider. Thirdly, the study period of two weeks was relatively short for efficacy assessment, and follow-up measures that might confirm the stability of the current effects were not included in this study. Taken together, findings of the present study need to be confirmed in further studies that overcome those limitations.

In conclusion, the present study provided an internet-based integrated intervention focusing on relaxation, self-care, and raising sense of security for COVID-19 patients with psychological distress. The preliminary results of this study demonstrated a pleasing effect of the internet-based integrated intervention on mild to moderate depression and anxiety symptoms.

\section{Contributors}

Ning WEI conducted the intervention and collected the clinical data. Bo-chao HUANG designed the integrated intervention. Shao-jia LU wrote the first draft of the manuscript. Jian-bo HU, Chan-chan HU, Jing-kai CHEN, and Jin-wen HUANG designed and completed the audio records. Xiao-yi ZHOU, Shu-guang LI, Zheng WANG, and Dan-dan WANG performed the statistics and analyses of the data. Yi XU and Shao-hua HU designed the study. All authors have read and approved the final manuscript, and therefore, have full access to all the data in the study and take responsibility for the integrity of the data and the accuracy of the data analysis.

\section{Compliance with ethics guidelines}

Ning WEI, Bo-chao HUANG, Shao-jia LU, Jian-bo HU, Xiao-yi ZHOU, Chan-chan HU, Jing-kai CHEN, Jin-wen HUANG, Shu-guang LI, Zheng WANG, Dan-dan WANG, Yi $\mathrm{XU}$, and Shao-hua HU declare that they have no conflict of interest.

All procedures followed were in accordance with the ethical standards of the responsible committee on human experimentation (institutional and national) and with the Helsinki Declaration of 1975, as revised in 2008 (5). Informed consent was obtained from all patients for being included in the study.

\section{References}

Andersson G, Cuijpers P, Carlbring P, et al., 2014. Guided internet-based vs. face-to-face cognitive behavior therapy for psychiatric and somatic disorders: a systematic review and meta-analysis. World Psychiatry, 13(3):288-295. https://doi.org/10.1002/wps.20151

Chua SE, Cheung V, McAlonan GM, et al., 2004. Stress and psychological impact on SARS patients during the outbreak. Can J Psychiatry, 49(6):385-390. https://doi.org/10.1177/070674370404900607

Duan L, Zhu G, 2020. Psychological interventions for people affected by the COVID-19 epidemic. Lancet Psychiatry, online.

https://doi.org/10.1016/S2215-0366(20)30073-0

Hamilton M, 1959. The assessment of anxiety states by rating. 
Br J Med Psychol, 32(1):50-55.

https://doi.org/10.1111/j.2044-8341.1959.tb00467.x

Hamilton M, 1967. Development of a rating scale for primary depressive illness. Br J Soc Clin Psychol, 6(4):278-296. https://doi.org/10.1111/j.2044-8260.1967.tb00530.x

Kroenke K, Spitzer RL, Williams JB, 2001. The PHQ-9: validity of a brief depression severity measure. J Gen Intern Med, 16(9):606-613.

https://doi.org/10.1046/j.1525-1497.2001.016009606.x

Liu S, Yang LL, Zhang CX, et al., 2020. Online mental health services in China during the COVID-19 outbreak. Lancet Psychiatry, online. https://doi.org/10.1016/S2215-0366(20)30077-8

Schröder J, Berger T, Westermann S, et al., 2016. Internet interventions for depression: new developments. Dialogues Clin Neurosci, 18(2):203-212.

Sherifali D, Ali MU, Ploeg J, et al., 2018. Impact of internetbased interventions on caregiver mental health: systematic review and meta-analysis. J Med Internet Res, 20(7): e10668. https://doi.org/10.2196/10668

Spitzer RL, Kroenke K, Williams JBW, et al., 2006. A brief measure for assessing generalized anxiety disorder: the GAD-7. Arch Intern Med, 166(10):1092-1097. https://doi.org/10.1001/archinte.166.10.1092

Tsang HWH, Scudds RJ, Chan EYL, 2004. Psychosocial impact of SARS. Emerg Infect Dis, 10(7):1326-1327. https://doi.org/10.3201/eid1007.040090

van der Houwen K, Schut H, van den Bout J, et al., 2010. The efficacy of a brief internet-based self-help intervention for the bereaved. Behav Res Ther, 48(5):359-367. https://doi.org/10.1016/j.brat.2009.12.009

Wu KK, Chan SK, Ma TM, 2005. Posttraumatic stress after SARS. Emerg Infect Dis, 11(8):1297-1300. https://doi.org/10.3201/eid1108.041083

Xiang YT, Yang Y, Li W, et al., 2020. Timely mental health care for the 2019 novel coronavirus outbreak is urgently needed. Lancet Psychiatry, 7(3):228-229.
https://doi.org/10.1016/S2215-0366(20)30046-8

Xinhua Net, 2020. Xinhua Headlines: a race against time! Wuhan battles coronavirus. http://www.xinhuanet.com/ english/2020-01/30/c_138743900.htm [Accessed on Mar. $12,2020]$.

Zhu N, Zhang DY, Wang WL, et al., 2020. A novel coronavirus from patients with pneumonia in China, 2019. $N$ Engl J Med, 382(2):727-733.

https://doi.org/10.1056/NEJMoa2001017

\section{中文概要}

\section{题 目: 基于网络的整合心理干预对新冠肺炎患者抑郁焦 虑症状的改善作用}

目 的: 探讨基于网络的整合心理干预对新冠肺炎患者抑 郁焦虑症状的有效性。

创新点: 针对大规模公共卫生事件的患者, 首次采用基于 网络的整合心理干预技术开展抑郁焦虑症状的 治疗。这不仅能够让隔离患者更便捷地获得心理 干预, 而且减少了心理医生的感染暴露机会。

方 法: 纳入 26 例伴有抑郁焦虑症状的新冠肺炎患者, 随机分为干预组和对照组。干预组给予基于网络 的整合心理干预, 患者在医生指导下, 通过智能 手机聆听音频材料, 并接受整合心理干预, 包括 放松训练、正念治疗、“安全地” 技术和蝴蝶拥 抱法; 对照组则给予支持性心理治疗。通过 HAMD-17 与 HAMA 量表评估患者的抑郁焦虑水 平。我们发现在 1 周末及 2 周末干预组患者 HAMD-17 与 HAMA 减分均明显优于对照组。

结 论: 基于网络的整合心理干预可有效改善伴有抑郁焦 虑症状的新冠肺炎患者的情绪症状, 且对隔离患者 来说更便捷, 也减少了心理医生的感染暴露机会。

关键词: 新冠肺炎; 基于网络的整合心理干预; 抑郁; 焦虑 